\title{
AlgaGEM - a genome-scale metabolic reconstruction of algae based on the Chlamydomonas reinhardtii genome
}

\author{
Cristiana Gomes de Oliveira Dal'Molin", Lake-Ee Quek, Robin W Palfreyman, Lars K Nielsen
}

From 6th International Conference of the Brazilian Association for Bioinformatics and Computational Biology (X-meeting 2010)

Ouro Preto, Brazil. 15-18 November 2010

\begin{abstract}
Background: Microalgae have the potential to deliver biofuels without the associated competition for land resources. In order to realise the rates and titres necessary for commercial production, however, system-level metabolic engineering will be required. Genome scale metabolic reconstructions have revolutionized microbial metabolic engineering and are used routinely for in silico analysis and design. While genome scale metabolic reconstructions have been developed for many prokaryotes and model eukaryotes, the application to less well characterized eukaryotes such as algae is challenging not at least due to a lack of compartmentalization data.

Results: We have developed a genome-scale metabolic network model (named AlgaGEM) covering the metabolism for a compartmentalized algae cell based on the Chlamydomonas reinhardtii genome. AlgaGEM is a comprehensive literature-based genome scale metabolic reconstruction that accounts for the functions of 866 unique ORFs, 1862 metabolites, 2249 gene-enzyme-reaction-association entries, and 1725 unique reactions. The reconstruction was compartmentalized into the cytoplasm, mitochondrion, plastid and microbody using available data for algae complemented with compartmentalisation data for Arabidopsis thaliana. AlgaGEM describes a functional primary metabolism of Chlamydomonas and significantly predicts distinct algal behaviours such as the catabolism or secretion rather than recycling of phosphoglycolate in photorespiration. AlgaGEM was validated through the simulation of growth and algae metabolic functions inferred from literature. Using efficient resource utilisation as the optimality criterion, AlgaGEM predicted observed metabolic effects under autotrophic, heterotrophic and mixotrophic conditions. AlgaGEM predicts increased hydrogen production when cyclic electron flow is disrupted as seen in a high producing mutant derived from mutational studies. The model also predicted the physiological pathway for $\mathrm{H}_{2}$ production and identified new targets to further improve $\mathrm{H}_{2}$ yield.
\end{abstract}

Conclusions: AlgaGEM is a viable and comprehensive framework for in silico functional analysis and can be used to derive new, non-trivial hypotheses for exploring this metabolically versatile organism. Flux balance analysis can be used to identify bottlenecks and new targets to metabolically engineer microalgae for production of biofuels.

\footnotetext{
* Correspondence: c.gomesdeoliveira@uq.edu.au

Australian Institute for Bioengineering and Nanotechnology, The University

of Queensland, Brisbane Qld 4072 Australia
} 


\section{Background}

Microalgae are receiving increased attention as the search for sustainable and profitable biofuel feedstocks progresses. Algae-derived hydrogen, methane, triacylglycerols, and ethanol could all serve as potential biofuels [1-3], but many challenges remain to be addressed [4,5]. In order to realise the rates and titres necessary for commercial production, system-level metabolic engineering will be required [6].

In modern, system-level microbial metabolic engineering, genome scale metabolic reconstructions (GEMs) are used to integrate and analyse large 'omics datasets as well as to evaluate designs in silico. A GEM maps annotated metabolic genes and proteins to reactions based on the current best understanding of a given organism. A growing number of metabolic engineering studies have demonstrated the use of well-curated GEMs to generate strain designs that are neither intuitive nor obvious [7-12].

Currently there is no genome scale reconstruction available for algae. The first attempt to reconstruct a large metabolic reconstruction of algae (based on Chlamydomonas reinhardtii) featured 484 reactions and 458 metabolites located in the chloroplast, cytosol and mitochondria [13]. An independent model featured 259 reactions and 267 metabolites localized to the cytosol, mitochondria, chloroplast, glyoxysome, and flagellum [14]. Despite the importance of these models, curation of cellular compartmentalization and genomic information was limited to central metabolism. Furthermore, in their current format, such models do not allow for the integration of other omics data (proteome, transcriptome and metabolome) for a system-level assessment of C. reinhardtii. For this, a full GEM is required.

GEMs have been developed for several model eukaryotes: yeast [15], mouse [16], human [17] and Arabidopsis [18]. For algae and other less extensively studied eukaryotes, a major challenge is the scarcity of data regarding compartmentalisation. An approach to overcome this shortfall in information is to use the compartmentalisation data for related organisms (here Arabidopsis), where no biochemical data for algae exists. We recently used this approach for the metabolic reconstruction of GEMs for the C4 grasses, maize, sorghum and sugarcane, and the resultant model was able to predict differential protein expression between mesophyll and bundle sheath, a unique C4 phenomenon [19]. The metabolism of single-cellular C. reinhardtii, however, has several features distinct from plants, including the presence of fermentative pathways, an inability to utilize sugars and a distinct mechanism for photorespiration.

In this paper, we develop the first compartmentalized, genome-scale model of algae metabolism (named AlgaGEM) based on the $C$. reinhardtii genome and a comprehensive evaluation of biochemical evidence found in literature complemented with missing compartmentalisation data derived from the GEM for Arabidopsis, AraGEM [18]. AlgaGEM captures the unique algae phenotypes, identifies pathways known to be important during anaerobic growth and accurately predicts the effect of a known mutation on hydrogen production. The success highlights the potential of using chimeric models to access the immensely powerful tools available for analysing GEMs, when working with biochemically less characterized eukaryotes.

\section{Methods}

\section{Genome-scale metabolic reconstruction and functional annotation}

The genome-scale reconstruction process was adapted from the method applied to the GEM of Mus musculus [20], Arabidopsis (AraGEM) [18], maize sorghum and sugarcane (C4GEM)[19] (Figure 1). The core of the algae genome-scale model (AlgaGEM ver 1.0) was reconstructed from the $C$. reinhardtii gene and reaction database publicly available from Kyoto Encyclopedia of Genes and Genomes (KEGG) (Release 54.1, May 1, 2010) [21]. The reconstruction retained all reaction attributes from KEGG, including unique reactions, compound IDs and reaction reversibilities. In a few cases, KEGG uses multiple labels to describe the same compound, e.g., the use of non-specific and specific references to sugar stereoisomers (e.g., D-Glucose versus $\alpha$ D-Glucose). Each such multiplicity was resolved as described previously $[18,20]$. Another KEGG related issue addressed is the presence of unbalanced reactions, typically for (i) the synthesis or breakdown of polymers (e.g., DNA + nucleotide = DNA), (ii) use of generic groups " $R$ " and (iii) the consumption or production of $\mathrm{H}_{2} \mathrm{O}, \mathrm{H}^{+}$, and redox equivalents (e.g., $\left.\mathrm{NAD}(\mathrm{P}) \mathrm{H}\right)$. In AlgaGEM, polymers are described in the form of their corresponding monomers and the use of the generic atom " $R$ " was avoided.

In order to capture metabolism accurately, AlgaGEM was compartmentalised into cytosol, mitochondrion, plastid and microbody (functionally equivalent to peroxisomes in plants). The available metabolic pathways databases like KEGG [22] and BioCyc [23] do not capture the compartmentalization of metabolism in eukaryotes and we first attempted to use a protein subcellular localization predictive tool (WPsort, http://wolfpsort.org/)[24]. The predictions, however, were ambiguous or inconsistent across the organelles and did not produce a biochemically functional network. Instead, compartmentalisation was performed based on (a) experimental evidence whenever available from the literature [25-36] (see additional file 1; Table S1) and available on line resources (Table 1) or (b) localisation of enzyme homologs in Arabidopsis (AraGEM) [18]. 


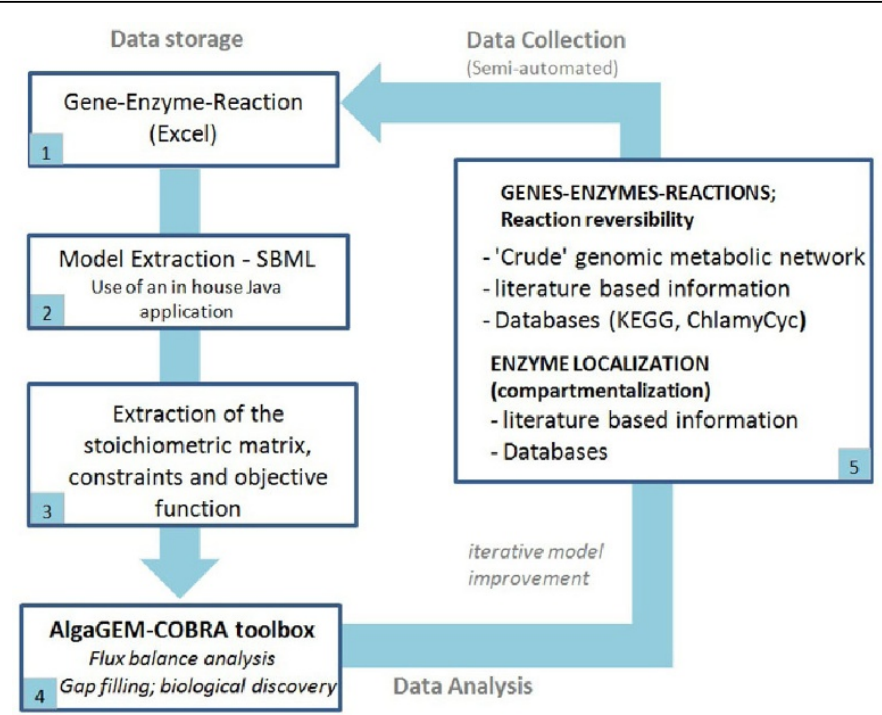

GENOME ANNOTATION

Chlamydomonas reinhardtii

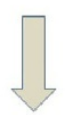

MANUAL CURATION

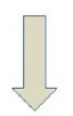

FUNCTIONAL VAUDATION

(physiological characteristics)

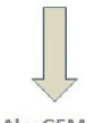

AlgaGEM

Figure 1 The process for genome-scale model reconstruction. (1) Metabolic properties including associations between genes, enzymes and reactions were extracted from the genomic metabolic databases, stored and curated in an Excel spread-sheet; (2) a 2D reaction centric SBML representation was generated using an in house java application. (3) The stoichiometric matrix, as well as reversibility constraints and the objective function were extracted from the SBML; (4) the relevant linear programming problems were solved using the COBRA toolbox [37]. (5) The model was refined in an iterative process, accessing the best available information in the literature and online data sources to achieve metabolic functionality.

Enzymes with no experimental data and no homolog found in Arabidopsis (e.g., fermentative reactions) were assigned to the cytosol per default. Transport reactions between cytoplasm and extracellular space and between cytoplasm and organelles are poorly annotated and were added manually based on transport reactions reported in the literature.
AlgaGEM was compiled and curated in Excel (Microsoft Corporation) for ease of annotation and commenting (Figure 1). From this gene centric database, a 2D reaction centric SBML (System Biology Markup Language, http://www.sbml.org) representation was generated using an in house Java (Oracle Corporation) application. As there is currently no specific element in SBML

Table 1 Online resources for the reconstruction of the metabolic network of Chlamydomonas reinhardtii

Database
Genome Database
DOE Joint Genome Institute (JGl); Chlamydomonas re
An Online Informatics Resource for Chlamydomonas
Pathway Databases
Kyoto Encyclopedia of Genes and Genomes (KEGG)
ChlamyCyc
Metacyc
ExPASy Biochemical Pathways

ExPASy Biochemical Pathways
ExPASy Enzyme Database

\section{Enzyme/Protein Localization and others Databases*}

AraPerox (Arabidopsis Protein from Plant Peroxisomes)

SUBA (Arabidopsis subcellular database)

PPDB (Plant proteome database)

UniproKB/SwissProt

Transport DB
Link

http://genome.jgi-psf.org/Chlre4/Chlre4.home.html

http://www.chlamy.org/chlamydb.html

http://www.genome.jp/kegg/pathway.html

http://chlamyto.mpimp-golm.mpg.de/chlamycyc/index.jsp

http://metacyc.org/

http://www.expasy.ch/cgi-bin/search-biochem-index

http://ca.expasy.org/enzyme/

http://www.brenda-enzymes.info/

http://www.araperox.uni-goettingen.de/

http://www.plantenergy.uwa.edu.au/applications/suba2/index.php

http://ppdb.tc.cornell.edu/default.aspx

http://ca.expasy.org/sprot/relnotes/relstat.html

http://www.membranetransport.org/

*Manual curation based on literature and homology sequence. 
allocated to store the gene-protein-reaction associations (e.g. splice-variants, isozymes, protein complexes), these were added as notes to the reaction elements. Constraint-based reconstruction and analysis was performed using COBRA toolbox (http://opencobra.sourceforge.net/) [37]; a set of MATLAB scripts for constraintbased modelling run from within the MATLAB environment (Version 7.3, The MathWorks, Inc.). Simulated flux distributions were visualized on a metabolic flux map (for the visualization of overall changes in the central metabolism of a compartmentalized algal cell) drawn in Excel.

AlgaGEM was evaluated for its ability to produce major biomass components and cofactors (Table 2) under autotrophic (photons as energy source, $\mathrm{CO}_{2}$ as carbon source and nitrate or ammonia as nitrogen source), heterotrophic (acetate as carbon source, nitrate and/or ammonia and/or amino acids as nitrogen sources) and mixotrophic (photons as energy source, $\mathrm{CO}_{2}$ and acetate as carbon sources and nitrate or ammonia as nitrogen source) conditions. For each biomass component in Table 2 and each growth condition, the following linear programming problem was formulated and solved

$$
\begin{array}{ll}
\text { maximise } & v_{i} \\
\text { subject to } & \mathbf{S v}=\mathbf{0} \\
& \mathbf{v}_{\text {min }} \leq \mathbf{v} \leq \mathbf{v}_{\text {max }}
\end{array}
$$

where $v_{\mathrm{i}}$ is the corresponding biomass drain reaction. Where the maximum production rate of a biomass component was zero, gap analysis was performed. Some gaps were readily filled based on inspection of the corresponding pathways in KEGG, ChlamyCyc [38] and other available databases (Table 1). Others, such as inconsistent irreversibility constraints, stoichiometry errors, compound names, compartmentalization errors or missing transporters, required sequential tracing through the model to identify breakpoints and careful evaluation of the possible causes.

Once network gaps were closed, the individual biomass accumulation terms were combined into an overall biomass synthesis equation, with the appropriate coefficients assigned to each precursor to define the composition of biomass. The overall biomass synthesis equation depends on growth conditions and was designed to represent autotrophic, heterotrophic and mixotrophic conditions based on literature data [13]. Trace elements were not included in the biomass equation, since their contribution to overall flux is trivial.

\section{Model simulations}

After curation, AlgaGEM was used as a framework to test cell optimality and maximum bioproduct performance under different conditions. Constraints were applied and flux balance analysis was performed in MATLAB using COBRA Toolbox [37]. The COBRA files can be downloaded from the additional files (see additional file 2; AlgaGEM-COBRA). The folders include the SBML file and the respective constraints to represent each metabolic scenario. The minimum set of constraints imposed to represent the different growth conditions are shown in Table 3.

\section{Minimize energy and carbon source}

The final model was evaluated through the estimation of the flux distributions in three growth conditions: autotrophic, heterotrophic and mixotrophic. The flux distributions were determined using linear programming

$$
\begin{array}{ll}
\text { minimize } & \text { photon or acetate utilization } \\
\text { subject to } & \mathbf{S v}=\mathbf{0} \\
& \mathbf{v}_{\text {biomass }}=\mathbf{b} \\
& \mathbf{v}_{\min } \leq \mathbf{v} \leq \mathbf{v}_{\max }
\end{array}
$$

i.e., the distributions that minimize the use of the key energy substrate (photons or acetate), while achieving a specified growth rate.

\section{Maximize bio-product: $\mathrm{H}_{2}$}

We also used the model to test the network capacity to maximize $\mathrm{H}_{2}$ under different growth conditions.

$$
\begin{array}{ll}
\text { maximize } & \mathrm{H}_{2} \text { synthesis } \\
\text { subject to } & \mathrm{Sv}=\mathbf{0} \\
& \mathbf{v}_{\text {biomass }}=\mathbf{b} \\
& \mathbf{v}_{\min } \leq \mathbf{v} \leq \mathbf{v}_{\text {max }}
\end{array}
$$

Table 2 List of biomass components

\begin{tabular}{ll}
\hline $\begin{array}{l}\text { Carbohydrates and } \\
\text { sugars }\end{array}$ & Starch, sucrose, fructose, glucose, maltose \\
\hline $\begin{array}{l}\text { Protein (amino acids) } \\
\text { Alanine, arginine, aspartate, asparagine, cystein, lysine, leucine, isoleucine, glutamate, glutamine, histidine, methionine, } \\
\text { phenylalanine, proline, serine, tyrosine, tryptophan, valine }\end{array}$ \\
$\begin{array}{l}\text { Nucleotides } \\
\begin{array}{l}\text { Fatty acids } \\
\text { Vitamins and }\end{array} \\
\text { cofactors }\end{array}$ \\
\hline
\end{tabular}


Table 3 Minimal set of constraints imposed to represent different growth condition

\begin{tabular}{lccc}
\hline $\begin{array}{l}\text { Inputs, outputs and } \\
\text { constraints }\end{array}$ & Autotrophic & Heterotrophic & Mixotrophic \\
\hline C source: $\mathrm{CO}_{2}$ uptake & + & - & + \\
$\begin{array}{l}\text { C source: Acetate uptake } \\
\text { Photons uptake (free flux) }\end{array}$ & - & + & + \\
$\begin{array}{l}\text { Optimization 1: minimize } \\
\text { uptake of }\end{array}$ & Photons & Acetate & Photons \\
$\begin{array}{l}\text { Optimization 2: maximize } \\
\text { product }\end{array}$ & $\mathrm{H}_{2}$ & $\mathrm{H}_{2}$ & $\mathrm{H}_{2}$ \\
Biomass rate (fixed) * & $0.059 \mathrm{~h}^{-1}$ & $0.035 \mathrm{~h}^{-1}$ & $0.066 \mathrm{~h}^{-1}$ \\
\hline
\end{tabular}

*Biomass rate and biomass equation was used for each growth regime, based on measurements found in the literature [13]

i.e., the flux distributions that maximize $\mathrm{H}_{2}$ synthesis, while achieving a specified growth rate under autotrophic, mixotrophic or heterotrophic condition.

\section{Results and discussion}

\section{Characteristics of the reconstructed network}

Genome-scale metabolic models bridge the gap between genome-derived biochemical information and metabolic phenotypes in a structured manner. The genome-scale reconstruction (AlgaGEM, Ver 1.0) contains 2249 genereaction association entries, 1725 unique reactions and 1869 metabolites distributed across 4 cellular compartments (Table 4). The active scope of the AlgaGEM includes glycolysis (plastidic and cytosolic), the pentose phosphate pathway (PPP) (plastidic and cytosolic), tricarboxylic acid cycle (TCA cycle), light and dark reactions (Calvin cycle), fatty acid synthesis, beta-oxidation, glyoxylate cycle, photorespiratory cycle and fermentative reactions. The current version of AlgaGEM has not been tested for its coverage of secondary metabolism and some alternative fermentative pathways that are not well understood or described at the subcellular level for algae.

Forty two (42) biomass drain equations describe the accumulation of carbohydrates, sugars, amino acids and fatty acids, representing the major biomass drains for an

Table 4 Characteristics of the reconstructed genomescale model (AlgaGEM)

\begin{tabular}{ll}
\hline Metabolic properties & Total \\
\hline Gene-reaction-association entries & 2249 \\
Unique metabolic reactions & 1725 \\
Unique ORFs & 866 \\
Metabolites & 1862 \\
Cellular compartments & 4 \\
Biomass drains & 39 \\
Intercellular transporters & 24 \\
Inter-organelle transporters & 79 \\
Gaps (non-enzymatic reactions) & 3 \\
\hline
\end{tabular}

algal cell (Table 2). At present, fatty acid biosynthesis is limited to palmitic acid biosynthesis in chloroplasts. The biosynthetic pathways of a limited number of vitamins and co-factors have been curated to date. Twenty-four (24) intercellular exchange reactions (cytoplasm-extracellular) have been included to describe the uptake of light (absorbed photons), and the uptake/secretion of inorganic compounds $\left(\mathrm{CO}_{2}, \mathrm{H}_{2} \mathrm{O}, \mathrm{HCO}_{3-} ; \mathrm{O}_{2}, \mathrm{NO}_{3}\right.$, $\mathrm{NH}_{3}, \mathrm{H}_{2} \mathrm{~S}, \mathrm{SO}_{4}{ }^{2-}, \mathrm{PO}_{4}{ }^{3-}$ ), translocation of fermentative products (like acetate, glycolate, lactate and ethanol), $\mathrm{H}_{2}$ and amino acids (glutamine, glutamate, aspartate, alanine and serine). Together with biomass drains (39), the intercellular exchangers define the broad physiological domain of the model, that is, the curated aspects of $C$. reinhardtii primary metabolism captured by AlgaGEM. Inter-organelle transporters were added based on the biochemistry information available for algae (see additional file 1; Table S1). When not available, we used information that supports inter-organelle transporters for Arabidopsis (E.g.; Transport DB, Table 1). A total of 79 inter-organelle transporters were introduced in the model to achieve metabolic functionality. Apart from nomenclature and cellular compartmentalization issues, only three additional reactions without gene associations (non-enzymatic steps) were added during model curation before the model was able to simulate growth in silico.

\section{AlgaGEM predicts phosphoglycolate catabolism in algae}

The reconstruction of metabolic models for eukaryotes is challenging due to the scarcity of biochemical information at the subcellular level required for cellular compartmentalisation. AlgaGEM covers our current understanding of metabolic functionality and connectivity through different organelles for $C$. reinhardtii. It does rely, however, on AraGEM [18] for the compartmentalisation of reactions for which no data exist for $C$. reinhardtii. Given that there is a substantial, natural overlap between AlgaGEM and AraGEM with approximately $85 \%$ of all reactions present in both models, it is important to establish that AlgaGEM indeed predicts algae behaviour rather than slightly modified Arabidopsis behaviour.

Heterotrophic growth in AlgaGEM differs from AraGEM in that the former can metabolize acetate and glycolate, but lacks glucose and sucrose transporters and is unable to utilize these carbon sources from the media [33]. Moreover, AlgaGEM has fermentative reactions and produces a range of fermentative products (like $\mathrm{H}_{2}$, glycolate, acetate, formate, lactate, etc). These differences are the direct result of added reactions and transporters.

A more interesting difference is the way algae and plants handle the RuBisCO oxygenation reaction. 
Assuming that plants have evolved to optimise photon efficiency, AraGEM accurately predicts that phosphoglycolyate is recycled using the classical photorespiration cycle involving reactions in plastids, peroxisomes and mitochondria [18]. Moreover, it accurately predicts that photon requirements increase by $30-40 \%$, if the ratio of oxygenation-to-carboxylation is 1:4.

Photorespiration of C. reinhardtii deviates from the classical plant photorespiration in that instead of oxidizing glycolate to glyoxylate via glycolate oxidase in peroxisomes, C. Reinhardtii and many other microalgae utilize glycolate in mitochondria $[25,39,40]$. In addition, because molecular $\mathrm{O}_{2}$ is not an electron acceptor for glycolate dehydrogenase, glycolate oxidation catalysed by this enzyme does not produce $\mathrm{H}_{2} \mathrm{O}_{2}$, so catalase should not be required for the photorespiration cycle in algae. Instead, glycolate dehydrogenase is expected to contribute electrons to the mitochondrial respiratory electron transport through reduction of ubiquinone pool [40]. AlgaGEM accurately predicts that algae will catabolise rather than recycle phosphoglycolate, if sufficient oxygen is available and energy is needed, or alternatively secrete glycolate to the environment, as has also been observed $[41,42]$. AlgaGEM predicts the physiological pathways used for $\mathrm{H}_{2}$
production in Chlamydomonas under dark condition

Although substantial insights regarding algal $\mathrm{H}_{2}$ production exist $[1,43]$, critical aspects regarding the hydrogenase activity remain unresolved and new advances are required to define more clearly the metabolic and enzymatic processes influencing algal $\mathrm{H}_{2}$ production. AlgaGEM was used to capture contrasts in the metabolism when $\mathrm{H}_{2}$ is maximized during heterotrophic conditions (dark). Firstly, flux distribution was calculated by linear programming assuming carbon efficiency and minimum acetate usage to maintain cell growth under dark condition (see optimality criterion in methods). Secondly, the calculated optimum uptake rate of acetate to maintain biomass growth under heterotrophic conditions was fixed and $\mathrm{H}_{2}$ production was maximized to find the main metabolic changes. Ultimately we maximized $\mathrm{H}_{2}$ production under different uptake rates of acetate. Figure 2 highlights in green the increased fluxes (more than $20 \%$ increased) through different cell compartments when $\mathrm{H}_{2}$ production is maximized. During dark periods and using acetate as the sole carbon source, acetate is assimilated and storage compounds like starch are produced through gluconeogenic conversion [44]. Fluxes are increased through the glyoxylate cycle in the microbody and mitochondrial reactions, where acetate is mainly metabolised (showed by red arrows). It is believed that acetate is primarily converted to acetyl CoA via the glyoxylate cycle [44]. The glyoxylate cycle can generate one molecule of succinate as a net product from two molecules of acetate. As shown in Figure 2, the model predicts that acetate assimilation proceeds along the same pathway as gluconeogenic fatty acid conversion in oil seed. Succinate produced in the glyoxylate cycle is transported from the microbody and converted to malate and oxaloacetate in the mitochondrion. Oxaloacetate is decarboxilated to phosphoenolpyruvate in the cytosol, and phosphoenolpyruvate is converted into sugars phosphates. Our simulations show increased flux through phosphoenolpyruvate transported to chloroplast, and subsequent conversion into sugars and starch. The blue arrows highlight flux increased through enzymes involved in $\mathrm{H}_{2}$ synthesis. These steps refers to chloroplastic glyceraldehyde 3-phosphate dehydrogenase which supply reducing power during glycolysis and subsequent oxidation of pyruvate in the chloroplast catalysed by pyruvate ferredoxin oxidoreductase (PFR1) yielding acetyl-CoA, reduced ferredoxin and $\mathrm{CO}_{2}$ and mediates the observed production of $\mathrm{H}_{2}$ in the dark $[34,45-47]$. These steps are also summarized in Figure 3 where red reactions represent catabolism of organic substrates (reducing power supply) and green reactions represent dark fermentation. Both of $C$. reinhardtii's [Fe]-hydrogenases, HydA1 and HydA2, catalyze $\mathrm{H}_{2}$ production using electrons from ferredoxin. In several species of anaerobic microbes the decarboxylation of pyruvate to acetyl-coA by PFR1 is linked to hydrogen production via the reduction of ferredoxin [34,45-47]. The simulated flux distribution highlights the physiological pathways used for $\mathrm{H}_{2}$ production in Chlamydomonas when growing under dark conditions, as reported in the literature [44][34,45-47]. The identification in $C$. reinhardtii of a putative PFR1 gene, evidence of its coexpression with HydA1 and HydA2 [46][44] and our flux analysis suggests fermentative carbon metabolism and dark, anaerobic $\mathrm{H}_{2}$ production may be linked via ferredoxin. This could explain the source of electrons in the dark, fermentative $\mathrm{H}_{2}$ production, a discovery with the potential to improve dark $\mathrm{H}_{2}$ conversion efficiency. The model suggests that over expression of PFR1 gene should improve $\mathrm{H}_{2}$ yield under dark conditions by supplying the electron donor to hydrogenase. Further experimental investigation is required to validate this hypothesis.

\section{AlgaGEM predicts increased hydrogen production when cyclic electron flow is disrupted under mixotrophic conditions}

C. Reinhardtii produces hydrogen under heterotrophic and mixotrophic conditions (Figure 3). The rates are generally low and metabolic engineering strategies are being explored to improve production rates. Mutational studies identified a high producing, state transition 


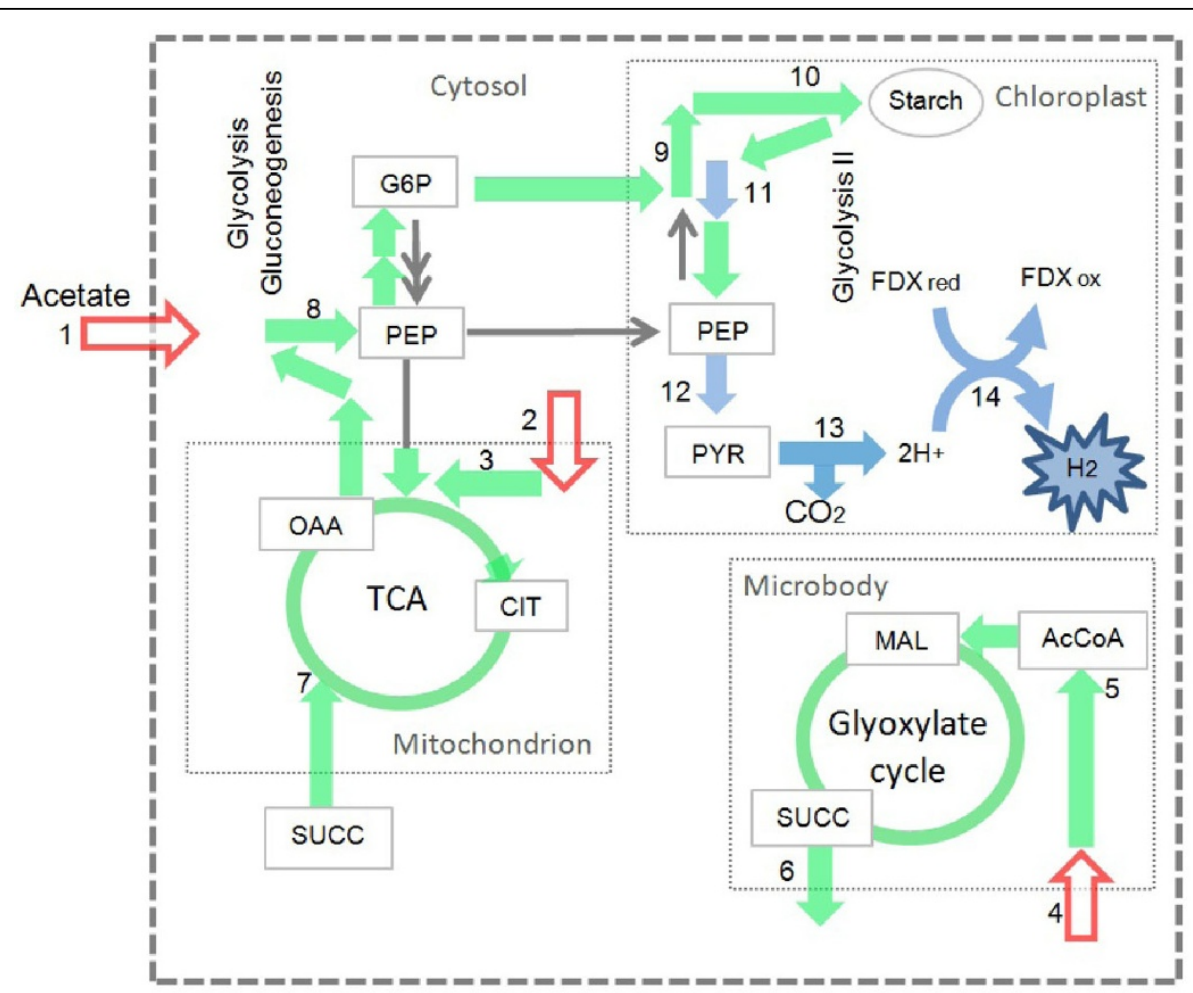

Figure 2 Increased fluxes through the physiological pathways used for $\mathrm{H}_{2}$ production in Chlamydomonas under dark condition. Acetate is assimilated (red arrows) and starch is produced through gluconeogenic conversion. Green arrows highlight increased fluxes through acetate metabolism, starch synthesis/degradation and the physiological pathways for $\mathrm{H}_{2}$ production in Chlamydomonas. The blue arrows are the increased fluxes through enzymes involved in $\mathrm{H}_{2}$ synthesis. Numbers represent the main enzymatic and transport reactions: (1) acetate assimilation; $(2,4)$ acetate transporter; $(3,5)$ acetyl-Coa synthetase; $(6,7)$ succinate transporter; $(8)$ phosphoenolpyruvate carboxykinase; $(9)$ phosphoglucomutase (10) 1,4-alpha-glucan branching enzyme; (11) glyceraldehyde 3-phosphate dehydrogenase; (12) pyruvate kinase; (13) pyruvate ferredoxin oxidoreductase (PFR1); (14) ferredoxin hydrogenase. GLU: glucose; PEP: Phosphoenolpyruvate; G6P: glucose 6-phosphate; SUCC: Succinatate; MAL: malate; CIT: citrate; OAA: oxaloacetate; FDX red: reduced ferredoxin; FDX ox: oxidized ferredoxin.

mutant, Stm6, which is blocked in state 1 photosynthesis and hence has greatly inhibited cyclic electron flow around photosystem I [48].

We used AlgaGEM to simulate the metabolic changes when $\mathrm{H}_{2}$ is produced under mixotrophic growth. We compared flux distributions for two optimality criteria: maximization of $\mathrm{H}_{2}$ (hypothetical $\mathrm{H}_{2}$ producer) and minimum energy resource usage to maintain normal growth rate (wild type). Flux simulations are provided in detail (see additional file 3; Table S2) and illustrated in Figure 4. Key changes are summarised in Table 5. Under mixotrophic conditions and constant illumination $\left(65 \mu \mathrm{E} / \mathrm{m}^{2} / \mathrm{s}\right)$, the model predicted about an 8 -fold increase in $\mathrm{H}_{2}$ production, if cyclic electron flow is inhibited. This is in line with observations for the stm6 mutant, which produced 5-13 fold more $\mathrm{H}_{2}$ compared to the wild type [48]. Cyclic electron flow is normally used to balance requirements for redox with requirements for ATP. When cyclic electron flow is inhibited, $\mathrm{H}_{2}$ production becomes a release valve for excess redox.
Also in line with observations for the Stm6 mutant, the model predicted increased linear electron flow and reduced mitochondrial TCA cycle activity (Table 5). Furthermore, the model predictions agreed with observations made in other studies regarding the correlation of hydrogen production with expression of various genes, including increased activity of reactions directly involved in hydrogen production (Fe-Hydrogenases, Ferredoxin-NADP+ reductase, glyceraldehyde -3-phosphate dehydrogenase, and pyruvate ferredoxin oxireductase) and a shift carbon assimilation through Calvin cycle/ $\mathrm{CO}_{2}$ assimilation to acetate assimilation (Table 5). The model also suggested a number of possible further targets for investigation, including gluconeogenesis, pentose phosphate pathway, beta oxidation, glyoxylate cycle and GS/GOGAT.

\section{Conclusions}

AlgaGEM is a curated, compartmentalized genome scale model of algal cell primary metabolism. Continued curation efforts will focus on closing gaps, especially in the 


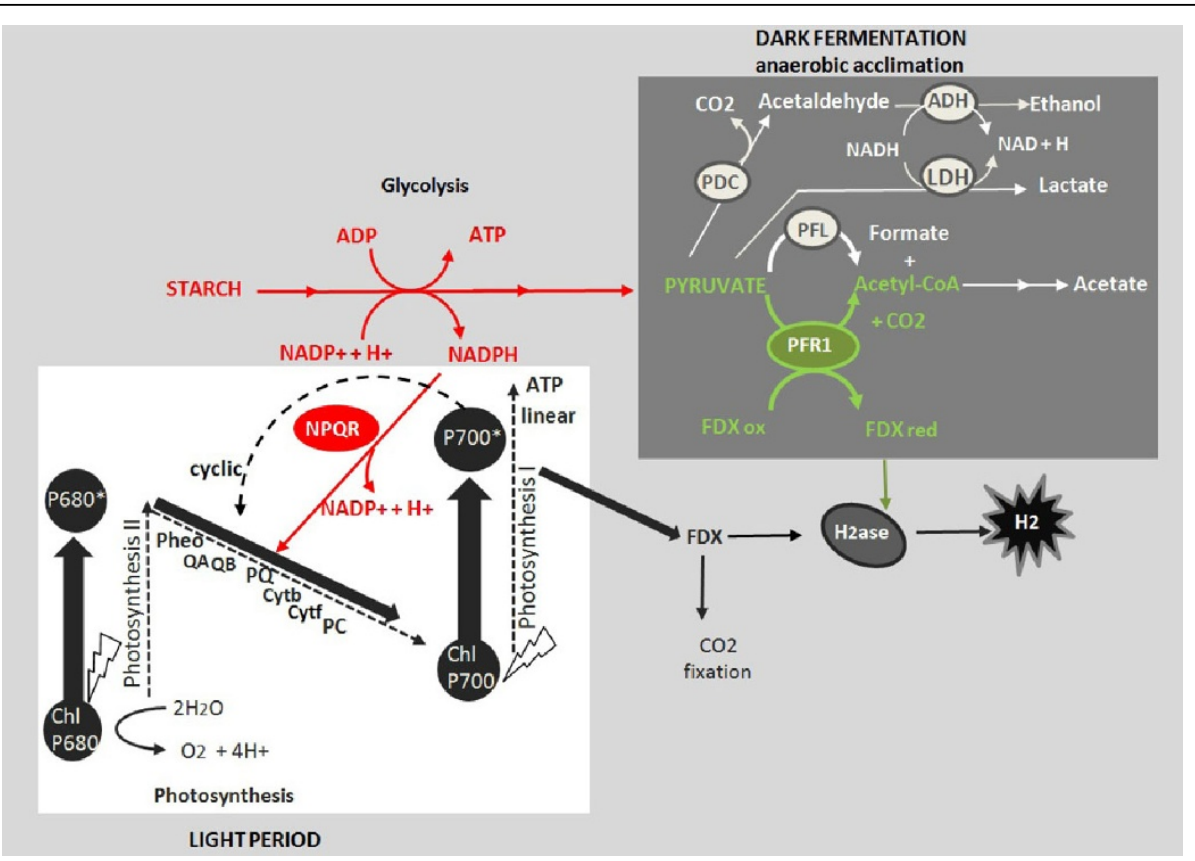

Figure 3 Simplified illustration of the physiological pathways used for $\mathrm{H}_{2}$ production in Chlamydomonas (Adapted from The Chlamydomonas Sourcebook [44]). The two photoproduction pathways involving PSII and PSI under the light period are showed in black. Electrons excited to higher energy (low potential) by PSI are able to reduce ferredoxin (FDX), the physiological electron donor to hydrogenase. Both the PSII-dependent and NPQR-dependent (red) pathways require reduction of the PQ pool and PSI activity. In the case of the PSIIindependent pathway (reactions in red), reducing power formed by the catabolism of organic substrates is used for reduction of the PQ pool. During dark fermentation the oxidation of pyruvate catalysed by PFR (green reactions) is used to reduce ferredoxin and putatively mediates the observed production of $\mathrm{H}_{2}$ in the dark. White reactions show the parallel main fermentative products from pyruvate, competing with $\mathrm{H}_{2}$. Dashed arrows show linear and cyclic electron flow.

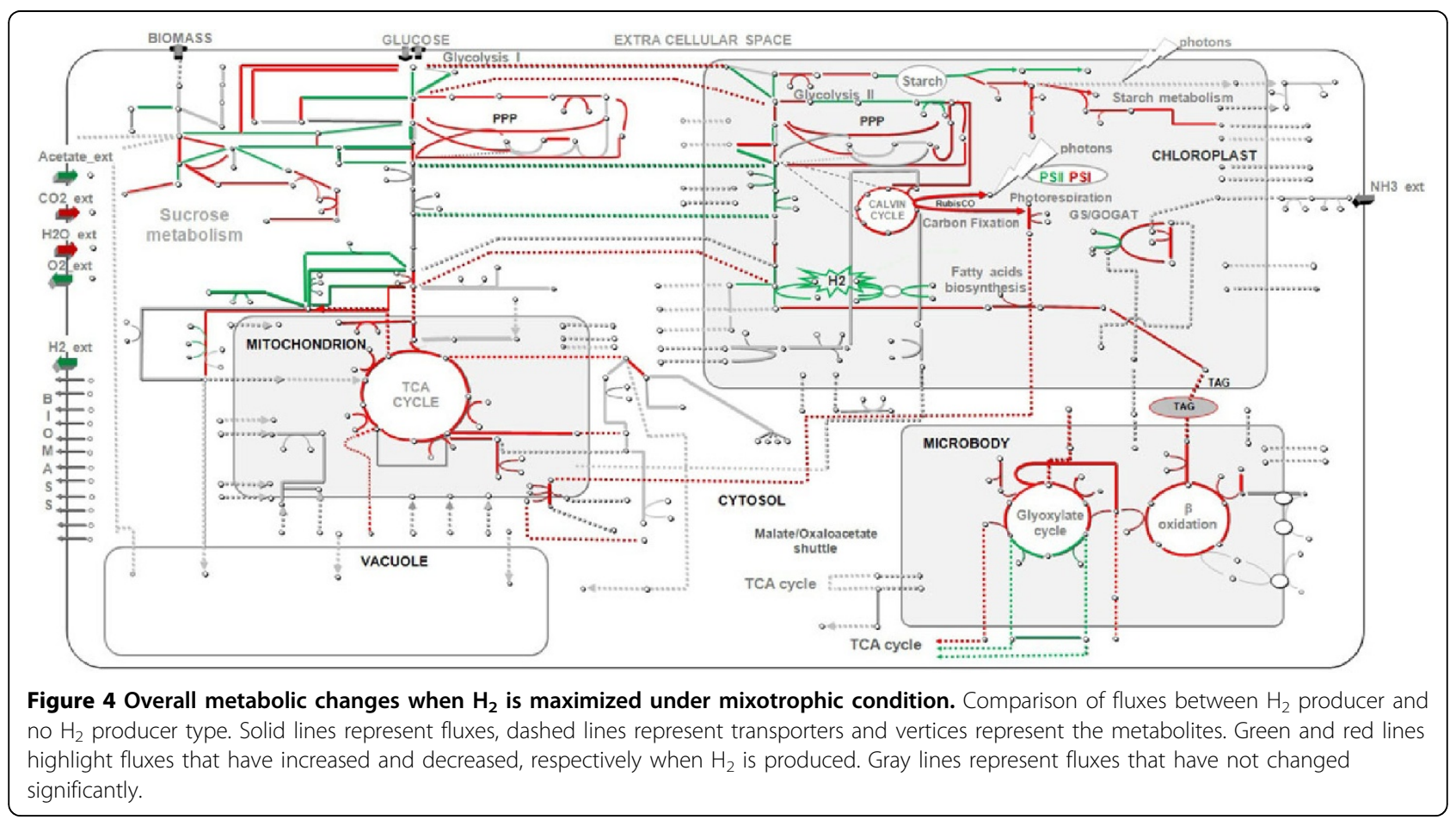


Table 5 Upregulation of key target enzymes when $\mathrm{H}_{2}$ is produced under mixotrophic conditions, highlighted by AlgaGEM

\begin{tabular}{lcc}
\hline Step reaction & $\begin{array}{c}\text { Regulation (up/ } \\
\text { down) }\end{array}$ & Reference $^{*}$ \\
\hline Cyclic electron flow (PSI) & - & {$[48]$} \\
Linear electron flow (PSII) & + & {$[48]$} \\
TCA cycle/mitochondria & - & {$[48]$} \\
Fe-Hydrogenases & + & {$[29,31,35,49-52]$} \\
Ferredoxin-NADP+ reductase (FNR) & + & {$[53]$} \\
Glyceraldehyde 3-P-dehydrogenase & + & {$[54-57]$} \\
Pyruvate ferredoxin oxireductase & + & {$[58-60]$} \\
Calvin Cycle/CO2 assimilation & - & {$[44]$} \\
Acetate assimilation & + & {$[44]$} \\
Gluconeogenesis (cytosolic) & + & Not found \\
Pentose phosphate pathway & - & Not found \\
(cytosolic) & & Not found \\
Pentose phosphate pathway & + & Not found \\
(plastidic) & - & Not found \\
Beta oxidation/glyoxylate cycle & + & Not found \\
glutamate synthase (ferredoxin); & &
\end{tabular}

* Results supported by evidences found in the literature. Not found: model highlights new targets in different pathways to improve $\mathrm{H}_{2}$ that need further experimental investigation for model validation.

secondary metabolism and alternative fermentative pathways (not well understood or described at organelle level for algae) and the resolution of gene product targeting where this is yet to be established. In its current version, the model covers the primary metabolism including some of the fermentative pathways. Importantly, while the model shares $85 \%$ of the reactions with AraGEM and while AraGEM was used to compartmentalise many genes, the model predicts distinct algal behaviours such as the catabolism or secretion rather than recycling of phosphoglycolate in photorespiration.

The use of AlgaGEM for in silico flux predictions illustrates the potential of using genome scale models to explore complex, compartmentalized networks and develop non-trivial hypotheses. The metabolic changes highlighted by AlgaGEM to increase $\mathrm{H}_{2}$ yield show agreement with evidence found in the literature and the model predicted the magnitude of change observed in a stage transition mutant, Stm6. Further experimental investigations are also suggested to test new targets. Such results support the potential use of this framework for algae metabolic engineering.

\section{Additional material}

Additional file 1: Supplemental material file. Table S1. List of interorganelle transporters and enzymes' localization based on biochemistry information.
Additional file 2: Supplemental material file. AlgaGEM-COBRA tool box. The folders include AraGEM (sbml format) and constraints to run flux balance analysis for different metabolic scenarios using COBRA tool box.

Additional file 3: Supplemental material file. Table S2. Metabolic flux simulations.

\section{Acknowledgments}

We thank Professor Ben Hankamer, Dr. lan Ross and Ms Winne Waudo (Institute for Molecular Biosciences/The University of Queensland) for the insightful discussion and for information about the $\mathrm{H}_{2}$ mutant (Stm6). This article has been published as part of BMC Genomics Volume 12 Supplement 4, 2011: Proceedings of the 6th International Conference of the Brazilian Association for Bioinformatics and Computational Biology ( $\mathrm{X}$ meeting 2010). The full contents of the supplement are available online at http://www.biomedcentral.com/1471-2164/12?issue=S4

\section{Authors' contributions}

CGO carried out the reconstruction and curation of AlgaGEM, performed in silico flux analysis and wrote the manuscript. LQ developed the method for the reconstruction and contributed in insightful discussions. RP worked on the data mining process and analysis. LKN conceived the study, performed analyses and revisions of the manuscript. All authors read and approve the final manuscript.

\section{Competing interests}

The authors declare that they have no competing interests.

Published: 22 December 2011

\section{References}

1. Kruse O, Hankamer B: Microalgal hydrogen production. Current Opinion in Biotechnology 2010, 21(3):238-243.

2. Rittmann BE: Opportunities for renewable bioenergy using microorganisms. Biotechnology and Bioengineering 2008, 100(2):203-212.

3. Sivakumar $\mathrm{G}$, et al: Bioethanol and biodiesel: Alternative liquid fuels for future generations. Engineering in Life Sciences 2010, 10(1):8-18.

4. Pienkos PT, Darzins A: The promise and challenges of microalgal-derived biofuels. Biofuels Bioproducts \& Biorefining-Biofpr 2009, 3(4):431-440.

5. Scott $S A$, et al: Biodiesel from algae: challenges and prospects. Current Opinion in Biotechnology 2010, 21(3):277-286.

6. Shimidt BJ, et al: Metabolic systems analysis to advance algal biotechnology. biotechnology Journal 2010, 5:660-667.

7. Tyo KEJ, Kocharin K, Nielsen J: Toward design-based engineering of industrial microbes. Current Opinion in Microbiology 2010, 13(3):255-262.

8. Tepper N, Shlomi T: Predicting metabolic engineering knockout strategies for chemical production: accounting for competing pathways. Bioinformatics 2010, 26(4):536-543.

9. Choi HS, et al: In Silico Identification of Gene Amplification Targets for Improvement of Lycopene Production. Applied and Environmental Microbiology 2010, 76(10):3097-3105.

10. Raman K, Chandra N: Flux balance analysis of biological systems: applications and challenges. Briefings in Bioinformatics 2009, 10(4):435-449.

11. Park JM, Kim TY, Lee SY: Constraints-based genome-scale metabolic simulation for systems metabolic engineering. Biotechnology Advances 2009, 27(6):979-988.

12. Melzer $\mathrm{G}$, et al: Flux Design: In silico design of cell factories based on correlation of pathway fluxes to desired properties. Bmc Systems Biology 2009, 3:120.

13. Boyle NR, Morgan J: Flux balance analysis of primary metabolism in Chlamydomonas reinhardtii. BMC Systems Biology 2009, 3(4):32.

14. Manichaikul A, et al: Metabolic network analysis integrated with transcript verification for sequenced genomes. Nat Methods 2009, 6(8):589-92

15. Duarte NC, Herrgard MJ, Palsson BO: Reconstruction and validation of Saccharomyces cerevisiae iND750, a fully compartmentalized genomescale metabolic model. Genome Research 2004, 14(7):1298-1309. 
16. Quek L, Nielsen LK: On the reconstruction of the Mus musculus genomescale metabolic network model. Genome Informatics 2008

17. Mo ML, Jamshidi N, Palsson BO: A genome-scale, constraint-based approach to systems biology of human metabolism. Molecular Biosystems 2007, 3(9):598-603.

18. Dal'Molin CGD, et al: AraGEM, a Genome-Scale Reconstruction of the Primary Metabolic Network in Arabidopsis. Plant Physiology 2010, 152(2):579-589.

19. Dal'Molin CG, et al: C4GEM, a genome-scale metabolic model to study C4 plant metabolism. Plant Physiol 2010, 154(4):1871-85.

20. Quek LE, Nielsen LK: On the Reconstruction of the Mus Musculus Genome-Scale Metabolic Network Model. Genome Informatics 2008, Vol 21 2008, 21:89-100.

21. Kanehisa M, et al: KEGG for representation and analysis of molecular networks involving diseases and drugs. Nucleic Acids Res 2010, 38(Database issue):D355-60.

22. Kanehisa $M$, et al: KEGG for linking genomes to life and the environment. Nucleic Acids Research 2008, 36:D480-D484

23. Krieger CJ, et al: MetaCyc: a multiorganism database of metabolic pathways and enzymes. Nucleic Acids Research 2004, 32:D438-D442.

24. Horton P, et al: WoLF PSORT: protein localization predictor. Nucleic Acids Res 2007, 35(Web Server issue):W585-7.

25. Nelson EB, Tolbert NE: Glycolate Dehydrogenase in Green Algae. Archives of Biochemistry and Biophysics 1970, 141(1):102-110.

26. Floyd GL, Salisbury JL: Glycolate Dehydrogenase in Primitive Green-Algae. American Journal of Botany 1977, 64(10):1294-1296.

27. Kato J, et al: Characterization of catalase from green algae Chlamydomonas reinhardtii. Journal of Plant Physiology 1997, 151(3):262-268.

28. Thyssen C, Schlichting R, Giersch C: The CO2-concentrating mechanism in the physiological context: lowering the $\mathrm{CO} 2$ supply diminishes culture growth and economises starch utilisation in Chlamydomonas reinhardtii. Planta 2001, 213(4):629-639.

29. Winkler $\mathrm{M}$, et al: [Fe]-hydrogenases in green algae: photo-fermentation and hydrogen evolution under sulfur deprivation. International Journal of Hydrogen Energy 2002, 27(11-12):1431-1439.

30. Nogales J, et al: Functional analysis and regulation of the malate synthase from Chlamydomonas reinhardtii. Planta 2004, 219(2):325-331.

31. Hackstein JHP: Eukaryotic Fe-hydrogenases - old eukaryotic heritage or adaptive acquisitions? Biochemical Society Transactions 2005, 33:47-50

32. Stabenau H, Winkler U: Glycolate metabolism in green algae. Physiologia Plantarum 2005, 123(3):235-245.

33. Doebbe A, et al: Functional integration of the HUP1 hexose symporter gene into the genome of C-reinhardtii: Impacts on biological $\mathrm{H}-2$ production. Journal of Biotechnology 2007, 131(1):27-33.

34. Ghirardi $\mathrm{ML}$, et al: Hydrogenases and hydrogen photoproduction in oxygenic photosynthetic organisms. Annual Review of Plant Biology 2007, 58:71-91.

35. Hemschemeier A, Jacobs J, Happe T: Biochemical and physiological characterization of the pyruvate formate-lyase Pfl1 of Chlamydomonas reinhardtii, a typically bacterial enzyme in a eukaryotic alga. Eukaryotic Cell 2008, 7(3):518-526.

36. Zhang ZH, et al: PMRD: plant microRNA database. Nucleic Acids Research 2010, 38:D806-D813.

37. Becker SA, et al: Quantitative prediction of cellular metabolism with constraint-based models: the COBRA Toolbox. Nat Protoc 2007, 2(3):727-38.

38. May $P$, et al: ChlamyCyc: an integrative systems biology database and web-portal for Chlamydomonas reinhardtii. Bmc Genomics 2009, 10:209.

39. Beezley BB, Gruber PJ, Frederick SE: Cytochemical localization of glycolate dehydrogenase in mitochondria of chlamydomonas. Plant Physiol 1976, 58(3):315-9.

40. Nakamura $Y$, et al: Disruption of the glycolate dehydrogenase gene in the high-CO2-requiring mutant HCR89 of Chlamydomonas reinhardtii. Canadian Journal of Botany-Revue Canadienne De Botanique 2005, 83(7):820-833.

41. Spencer KG, Togasaki RK: Limitations on the Utilization of Glycolate by Chlamydomonas reinhardtii. Plant Physiol 1981, 68(1):28-32.

42. Moroney JV, Wilson BJ, Tolbert NE: Glycolate Metabolism and Excretion by Chlamydomonas reinhardtii. Plant Physiol 1986, 82(3):821-6.
43. Chochois V, et al: Hydrogen Production in Chlamydomonas: Photosystem II-Dependent and -Independent Pathways Differ in Their Requirement for Starch Metabolism. Plant Physiology 2009, 151(2):631-640.

44. Stern DB, Harris EH, Witman G: The Chlamydomonas Sourcebook: Organellar and metabolic processes. San Diego, CA, USA.: Academic Press; 2009:2:1040.

45. Tolstygina IV, et al: Hydrogen Production by Photoautotrophic SulfurDeprived Chlamydomonas reinhardtii Pre-Grown and Incubated Under High Light. Biotechnology and Bioengineering 2009, 102(4):1055-1061.

46. Ghirardi ML, et al: Photobiological hydrogen-producing systems. Chemical Society Reviews 2009, 38(1):52-61.

47. Ikuta $Y$, et al: Hydrogen production by photosynthetic microorganisms. Biohydrogen 1998, 319-328.

48. Kruse $\mathrm{O}$, et al: Improved photobiological $\mathrm{H}-2$ production in engineered green algal cells. Journal of Biological Chemistry 2005, 280(40):34170-34177.

49. Forestier $M$, et al: Expression of two [Fe]-hydrogenases in Chlamydomonas reinhardtii under anaerobic conditions. European Journal of Biochemistry 2003, 270(13):2750-2758.

50. Happe T, Kaminski A: Differential regulation of the Fe-hydrogenase during anaerobic adaptation in the green alga Chlamydomonas reinhardtii. European Journal of Biochemistry 2002, 269(3):1022-1032.

51. Melis A, Seibert M, Happe T: Genomics of green algal hydrogen research. Photosynthesis Research 2004, 82(3):277-288.

52. Tamagnini $P$, et al: Hydrogenases and hydrogen metabolism of cyanobacteria. Microbiology and Molecular Biology Reviews 2002, 66(1):1-20.

53. Winkler $M$, et al: Characterization of the Key Step for Light-driven Hydrogen Evolution in Green Algae. Journal of Biological Chemistry 2009, 284(52):36620-36627

54. Ananyev G, Carrieri D, Dismukes GC: Optimization of metabolic capacity and flux through environmental cues to maximize hydrogen production by the cyanobacterium "Arthrospira (Spirulina) maxima". Applied and Environmental Microbiology 2008, 74(19):6102-6113.

55. Melis A: Photosynthetic $\mathrm{H}-2$ metabolism in Chlamydomonas reinhardtii (unicellular green algae). Planta 2007, 226(5):1075-1086.

56. Zhang LP, Happe T, Melis A: Biochemical and morphological characterization of sulfur-deprived and $\mathrm{H}$-2-producing Chlamydomonas reinhardtii (green alga). Planta 2002, 214(4):552-561.

57. Zhang $L$, Melis A: Probing green algal hydrogen production. Philos Trans $R$ Soc Lond B Biol Sci 2002, 357(1426):1499-1507.

58. Hemschemeier $A$, et al: Hydrogen production by Chlamydomonas reinhardtii: an elaborate interplay of electron sources and sinks. Planta 2008, 227(2):397-407.

59. Melis A, Happe T: Hydrogen production. Green algae as a source of energy. Plant Physiology 2001, 127(3):740-748.

60. Gaffron H, Rubin J: Fermentative and photochemical production of hydrogen in algae. J Gen Physiol 1942, 26(2):219-240.

doi:10.1186/1471-2164-12-S4-S5

Cite this article as: Gomes de Oliveira Dal'Molin et al:: AlgaGEM - a genome-scale metabolic reconstruction of algae based on the Chlamydomonas reinhardtii genome. BMC Genomics 2011 12(Suppl 4):S5.

\section{Submit your next manuscript to BioMed Central and take full advantage of:}

- Convenient online submission

- Thorough peer review

- No space constraints or color figure charges

- Immediate publication on acceptance

- Inclusion in PubMed, CAS, Scopus and Google Scholar

- Research which is freely available for redistribution

Submit your manuscript at www.biomedcentral.com/submit
C Biomed Central 